Foil-based atom chip for Bose-Einstein condensates

This article has been downloaded from IOPscience. Please scroll down to see the full text article.

2004 J. Phys. B: At. Mol. Opt. Phys. 372959

(http://iopscience.iop.org/0953-4075/37/14/009)

View the table of contents for this issue, or go to the journal homepage for more

Download details:

IP Address: 130.102.172.3

The article was downloaded on 08/06/2011 at 10:28

Please note that terms and conditions apply. 


\title{
Foil-based atom chip for Bose-Einstein condensates
}

\author{
C J Vale, B Upcroft, M J Davis, N R Heckenberg and \\ H Rubinsztein-Dunlop
}

Centre for Biophotonics and Laser Science, School of Physical Sciences, University of Queensland, Brisbane, Queensland 4072, Australia.

E-mail: vale@physics.uq.edu.au

Received 8 June 2004

Published 8 July 2004

Online at stacks.iop.org/JPhysB/37/2959

doi:10.1088/0953-4075/37/14/009

\begin{abstract}
We describe a novel method of fabricating atom chips that are well suited to the production and manipulation of atomic Bose-Einstein condensates. Our chip was created using a silver foil and simple micro-cutting techniques without the need for photolithography. It can sustain larger currents than conventional chips, and is compatible with the patterning of complex trapping potentials. A near pure Bose-Einstein condensate of $4 \times 10^{4}{ }^{87} \mathrm{Rb}$ atoms has been created in a magnetic microtrap formed by currents through wires on the chip. We have observed the fragmentation of atom clouds in close proximity to the silver conductors. The fragmentation has different characteristic features to those seen with copper conductors.
\end{abstract}

(Some figures in this article are in colour only in the electronic version)

\section{Introduction}

Bose-Einstein condensates (BECs) have become a valuable tool for probing many aspects of atomic and quantum physics. Recently, condensates have been produced in miniature magnetic traps formed by current-carrying wires patterned onto a substrate [1,2]. Such devices have become known as atom chips, and are finding wide application in efforts to coherently control matter for new atom-optical devices [3].

Atom chip BEC experiments have mostly been performed with chips fabricated using photolithographic techniques [4-6]. These chips have been highly successful and form the basis of a growing number of experiments. However, there are some limitations to chips produced in this manner. One is that the photoresist thickness and etching process constrain the maximum height of the conducting wires. Typical conductor thicknesses range from about $2 \mu \mathrm{m}$ for evaporatively or sputter-coated wires, up to $10 \mu \mathrm{m}$ for electroplated wires. The latter also appear to suffer from rough edges, leading to fragmentation of clouds near the 


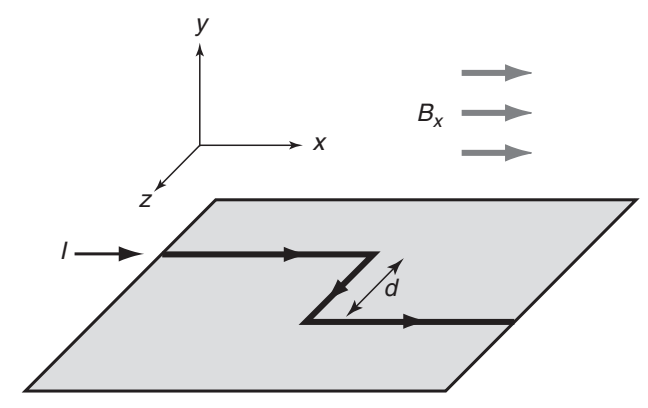

Figure 1. A miniature Ioffe-Pritchard trap on an atom chip. A Z-shaped wire and homogeneous bias field can produce a simple trap for weak-field seeking atoms above the centre of the middle section of wire, as described in the text.

surface [7]. A desirable feature of atom chips is that narrower wires result in tighter trapping potentials near the surface for a fixed current. However, the cross-sectional area (and hence the thickness) of a wire restricts the maximum current that can be sustained before it overheats and detaches from the surface. Current densities of order $10^{6} \mathrm{~A} \mathrm{~cm}^{-2}$ have been achieved on atom chips at currents of order $1 \mathrm{~A}$, but continuous operation at these current densities is fraught with danger. An elegant solution to this problem would be to use high- $T_{c}$ superconducting wires, although this introduces additional technical complexity. Thicker extruded wire [8] and machined copper conductors [9] have also been used to obtain higher currents, but these methods are not suited to sub-millimetre patterning.

In this paper we describe a method of fabricating atom chips with thick wires capable of sustaining currents of several amperes without overheating. We begin by reviewing magnetic microtraps and provide analytic expressions to describe the properties of the widely used Z-wire trap. We then describe the fabrication process for our chip and demonstrate its effectiveness by using it to produce a Bose-Einstein condensate of ${ }^{87} \mathrm{Rb}$. Finally, we look at cold atom clouds trapped close to the chip surface and observe fragmentation of the atomic density profile.

\section{Magnetic microtraps}

When an atom with a magnetic dipole moment, $\boldsymbol{\mu}$, is placed in a magnetic field, $\boldsymbol{B}$, it experiences an interaction potential $U=-\boldsymbol{\mu} \cdot \boldsymbol{B}$. If the projection of the magnetic moment onto the field remains constant during the atom's motion, the adiabatic condition is satisfied (i.e. the atom remains in the same magnetic substate, $m_{F}$ ). In such cases the potential is given by the scalar expression, $U=-m_{F} g_{F} \mu_{B} B$, where $g_{F}$ is the Lande g-factor, $\mu_{B}$ is the Bohr magneton and $B=|\boldsymbol{B}|$. Atoms whose magnetic moment aligns antiparallel to $\boldsymbol{B}$ are known as weak-field seekers, as their lowest energy state is at a minimum of $B$.

Several arrangements of wires have been proposed [10] and used in experiments to realize a variety of magnetic trapping geometries for weak-field seeking atoms [11,3]. A building block for all of these is the 'side guide', in which the combination of the field of a straight conducting wire and homogeneous bias field produces a two-dimensional, linear (to first order) trapping potential. A simple three-dimensional trap can be realized by bending a single wire into the shape of a $\mathrm{Z}$, as shown in figure 1 , where the top and bottom wire sections produce a confining potential in the longitudinal $(z)$ direction. 
The combination of a Z-shaped wire and transverse bias field, $B_{x}$, produces a IoffePritchard type trap with a nonzero minimum located above the centre of the middle section of wire. In the $x-y$ plane the trap minimum is where the field of the wire is exactly compensated by the bias field $B_{x}$. While the $x$ and $y$ components of the field go to zero here, the $z$-component, $B_{z}$, is nonzero out of the plane of the chip $(y=0)$, as the fields due to the two sections of current in the $x$-direction add constructively. Treating the wires as having an infinitesimal cross-section, the distance from the wire to the minimum is given by

$$
y_{0}=\frac{\mu_{0} I}{2 \pi B_{x}}
$$

where $\mu_{0}$ is the magnetic permeability of free space and $I$ is the current through the wire. The magnitude of the field gradient near the minimum $(x, y, z)=\left(0, y_{0}, 0\right)$ is equal in the radial ( $x$ and $y$ ) directions, and is given by

$$
\frac{\partial\left|B\left(0, y_{0}, 0\right)\right|}{\partial x}=\frac{\partial\left|B\left(0, y_{0}, 0\right)\right|}{\partial y}=\frac{\mu_{0} I}{2 \pi y_{0}^{2}}=\frac{B_{x}}{y_{0}} .
$$

In the axial $(z)$ direction the trapping potential is well described by half of the $z$-component of field produced by two infinite wires carrying current in the $x$-direction, located at $(y, z)=(0, \pm d / 2)$. The trapping potential is given by

$$
B_{z}(0, y, z)=\frac{\mu_{0} I}{4 \pi}\left(\frac{1}{(z-d / 2)^{2}+y^{2}}+\frac{1}{(z+d / 2)^{2}+y^{2}}\right),
$$

which is approximately harmonic at the centre with a curvature

$$
\frac{\partial^{2} B_{z}}{\partial z^{2}}=\frac{16 \mu_{0} I}{\pi} \frac{3 d^{2}-4 y^{2}}{d^{2}+4 y^{2}} .
$$

The residual offset field in the $z$-direction at the minimum of the trap is

$$
B_{z}\left(0, y_{0}, 0\right)=\frac{\mu_{0} I}{\pi} \frac{-2 y_{0}}{d^{2}+4 y_{0}^{2}}
$$

which can be partially compensated by an additional field along $-z$ to increase the transverse confinement.

Near the minimum $\left(0, y_{0}, 0\right)$, the trapping potential can be approximated as being ellipsoidal with the magnitude of the total field

$$
|B(r, z)|=\left[B_{z}\left(0, y_{0}, z\right)^{2}+\left(r \frac{\partial\left|B_{r}\right|}{\partial r}\right)^{2}\right]^{1 / 2},
$$

where $B_{z}\left(0, y_{0}, z\right)$ is given by equation 3 and $\partial\left|B_{r}\right| / \partial r$ by equation 2 .

\section{Atom chip}

Our atom chip was fabricated entirely in-house using materials and machinery readily available to typical laboratories. A schematic of the chip is shown in figure 2.

A $125 \mu \mathrm{m}$ silver foil (Goodfellow AG000360) was glued onto a $1 \mathrm{~mm}$ thick machinable ceramic (macor) substrate using EpoTek H77 ultra high vacuum (UHV) compatible epoxy. Silver was chosen as the conductive material as it has the lowest resistivity of all metals $(1.59 \mu \Omega \mathrm{cm})$, compared with gold $(2.2 \mu \Omega \mathrm{cm})$ and copper $(1.67 \mu \Omega \mathrm{cm})$. It is also highly 


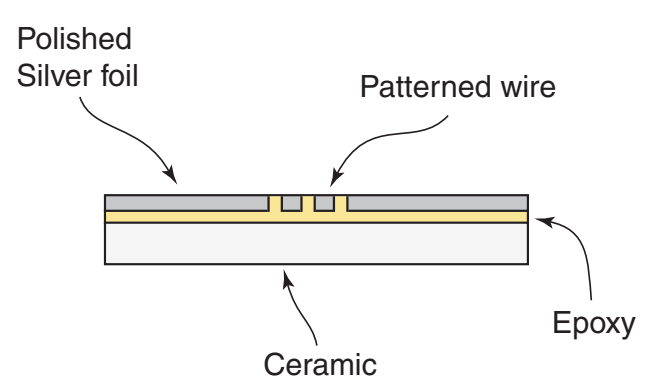

Figure 2. Schematic of the atom chip. A silver foil was glued onto a ceramic substrate using UHV compatible epoxy. The polished foil was approximately $90 \mu \mathrm{m}$ thick and the epoxy layer was about half this thickness. Insulating channels were cut into the foil with a micro-mechanical cutter and the channels were back-filled with epoxy.

reflective $(97 \%)$ to light resonant with the Rb D2 transition at $780 \mathrm{~nm}$. The epoxy adheres strongly to the ceramic, but less effectively to the silver. Improved bonding to the silver was achieved by roughening the surface to be glued with fine-grade sand paper. A $100 \mathrm{~nm}$ platinum layer was evaporatively coated onto the foil, which was then oxidized (with heat in air) before gluing. After curing the epoxy, the foil was polished to a mirror finish in three stages. Firstly, fine-grade abrasive paper held on a flat block was used under running water to take out any large $(>10 \mu \mathrm{m})$ ripples in the surface. Secondly, a range of finer grade abrasive cloths (Micromesh 1500-8000) were used down to a grain size of $3 \mu \mathrm{m}$. Finally, $3 \mu \mathrm{m}$ and $1 \mu \mathrm{m}$ grain size diamond polishing pastes (ProSciTech M23/3 and M23/1) were applied to the surface with a silk polishing cloth. These three polishing stages typically removed about $30-40 \mu \mathrm{m}$ of the silver, leaving a final conductor thickness of approximately $90 \mu \mathrm{m}$. The finished surface had very few scratches visible to the naked eye. A near-infrared laser beam was reflected from the finished surface and viewed several metres away without visible distortion. The power in the reflected beam was $95 \pm 2 \%$ of the incident power, in good agreement with the known reflectivity of silver at $780 \mathrm{~nm}$.

The polished chip was then ready to be machined. A computer numerically controlled (CNC) milling machine was used to cut insulating gaps into the silver foil using a $150 \mu \mathrm{m}$ diameter PCB cutting tool (LPKF $0.15 \mathrm{~mm}$ end mill, 107244). The wire patterns were programmed into the $\mathrm{CNC}$ mill and the cutting was performed in several runs, with the cut depth incremented by $20 \mu \mathrm{m}$ each run. The cutting tool spun at $10 \mathrm{krpm}$ and traced the pattern of the wires at a speed of $5 \mathrm{~mm} \mathrm{~min}^{-1}$. We found these parameters gave good results, but expect that higher rotation rates would be desirable. Deeper cutting increments (up to $50 \mu \mathrm{m}$ ) were also tested and no noticeable degradation of the cuts were observed. However, the tool was seen to wear very quickly if it penetrated the ceramic and so we were cautious with our increments. Small holes (1 mm diameter) were drilled through the foil, epoxy and macor near the edges of the chip, in the centre of the current connection pads.

After the pattern was cut, the resulting insulating channels were carefully backfilled with epoxy to provide additional mechanical support and heatsinking for the wires. Finally, the whole chip was repolished using the diamond pastes to remove any scratches or residues from the cutting process. The chip was cleaned with distilled water and methanol, before the electrical connections were assembled and the chip was placed in the vacuum chamber.

The robust nature of our chip allows simple yet secure electrical connections to the wires. The holes drilled through the connection pads accommodate $1 \mathrm{~mm}$ diameter screws. Copper 


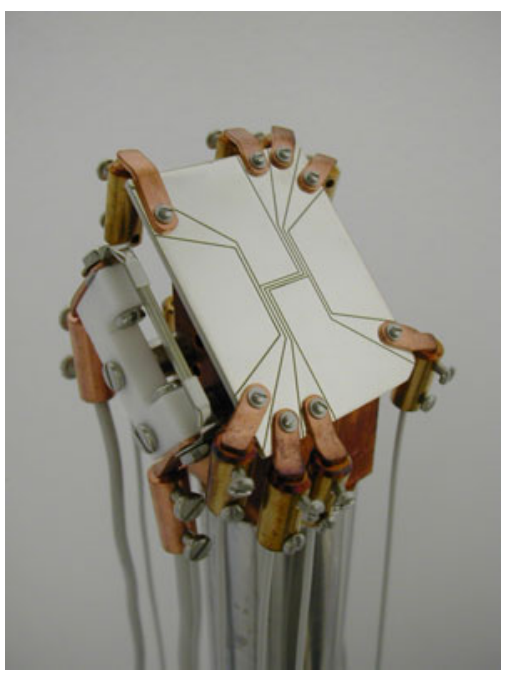

Figure 3. Photograph of the atom chip before being placed in the vacuum chamber. The electrical connections via the copper tabs are visible on the four corners of the chips. The rubidium dispenser can be seen on the left side of the chip mount.

tabs, also with holes drilled through them, were placed onto the connection pads and held in place by screws with a nut and sprung washer that prevented loosening during bake-out of the vacuum system. By contacting over a large area of the pad we ensure very low resistance connections and negligible heating. Figure 3 shows the completed chip with the electrical connections in place. The copper tabs are attached to barrel connectors, which in turn connect to thicker ceramic coated wires (Kurt J Lesker, CCWA10SI and CCWA20SI) that run to the electrical feedthroughs for the vacuum chamber. Also visible is the rubidium dispenser, attached to the side and recessed by a few millimetres from the silver surface of the chip.

We have tested the current-carrying capacity of our chip wires and found that individual wires can sustain currents of $8 \mathrm{~A}$ in vacuo for $10 \mathrm{~s}$ with the temperature remaining below $100{ }^{\circ} \mathrm{C}$. The temperature was evaluated by monitoring the resistance of the wire from outside the vacuum chamber. This necessarily includes the resistance of the connections and connecting wires - our estimate of the heating assumes that the increase in resistance observed was entirely due to heating of the chip wires. We therefore obtain an upper limit for the temperature increase of the chip. A nude ion gauge located approximately $30 \mathrm{~cm}$ from the chip could detect no increase of the pressure in the vacuum chamber at the level of $2 \times$ $10^{-11}$ mbar during these measurements. The current density in the wire was $5 \times 10^{4} \mathrm{~A} \mathrm{~cm}^{-2}$.

Figure 3 also shows the wires patterned onto the chip. These consist of two parallel Z-trap wires with a centre-to-centre separation of $2 a=0.4 \mathrm{~mm}$, and two additional endcap wires for varying the axial confinement. The parallel $\mathrm{Z}$-wires are capable of producing fields suitable for realizing the two-wire guide proposed in [12]. Assuming equal currents in the two wires, there are three possible configurations in the $x-y$ plane. These can be selected by the strength of the (dimensionless) bias field, $\beta=B_{x} / B_{0}$, where $B_{0}=\mu_{0} I / 2 \pi a$. The first configuration, with $\beta<1$, consists of two quadrupole traps in the $x=0$ plane, separated vertically by $2 a \sqrt{1-\beta^{2}} / \beta$. The second is a single hexapole trap which occurs at the critical bias field value $(\beta=1)$, with a minimum at $(x, y)=(0, a)$. The third configuration is for $\beta>1$ and produces two symmetric traps separated horizontally by $2 a \sqrt{\beta^{2}-1} / \beta$. 
In the axial direction there is only one trapping configuration, as the atoms are much further away from the two end-cap wire sections than the wire separation. The axial trap is well described by equations 3 and 4 for a single wire Z-trap carrying twice the current.

\section{Experiment}

We have used our atom chip to produce Bose-Einstein condensates of ${ }^{87} \mathrm{Rb}$ atoms. The experiment follows the usual stages for BEC production and the details of our procedure are described below.

Our vacuum chamber consists of a glass cell (made in our glass-blowing workshop) attached to a stainless steel vacuum chamber. We use a $751 \mathrm{~s}^{-1}$ ion pump and a nonevaporable getter to reach pressures below $2 \times 10^{-11}$ mbar. This was achieved by baking the chamber for two weeks at a temperature of $140{ }^{\circ} \mathrm{C}$ and pumping it with a turbo pump. The epoxy bonding the silver to the ceramic is rated to $150{ }^{\circ} \mathrm{C}$ which limits the bake-out temperature.

Rubidium vapour is obtained from a dispenser source which is pulsed with a current of 7.2 A for $12 \mathrm{~s}$. We collect $10^{8}$ atoms in a reflection magneto-optical trap (MOT) formed $4.5 \mathrm{~mm}$ from the surface of the chip. Trapping light is provided by a Toptica DLX $110400 \mathrm{~mW}$ external cavity diode laser system sent through a single mode fibre. It is tuned $15 \mathrm{MHz}$ below the $5 \mathrm{~S}_{1 / 2} F=2$ to $5 \mathrm{P}_{3 / 2} F^{\prime}=3$ transition in ${ }^{87} \mathrm{Rb}$. Four trapping beams enter the chamber, each with a power of $23 \pm 1 \mathrm{~mW}$ and $1 / \mathrm{e}^{2}$ diameter of approximately $25 \mathrm{~mm}$. The repump laser is a home-built external cavity diode laser system locked to the $F=1$ to $F^{\prime}=2$ transition. This laser is combined with the trapping laser on a polarizing beam splitter cube, and approximately $10 \mathrm{~mW}$ of repump light enters the chamber.

After the dispenser pulse, atoms are held in the MOT for a further $10 \mathrm{~s}$ to allow the vacuum to recover. At this distance from the surface the MOT does not appear to be seriously depleted by the imperfections in our mirror due to the insulating gaps. While we cannot make a direct comparison with a MOT with a near perfect mirror, our atom number $\left(10^{8}\right)$, temperature $(90 \mu \mathrm{K})$ and lifetime (40 s) are sufficient for producing condensates.

Next, the atoms are transferred to a compressed MOT with a magnetic field provided by a U-shaped wire centred $1.6 \mathrm{~mm}$ below the silver surface carrying a current of $20 \mathrm{~A}$, combined with a uniform transverse bias field of $12 \mathrm{G}$. This results in a spherical quadrupole field with gradients of $35 \mathrm{G} \mathrm{cm}^{-1}$ radially and $5 \mathrm{G} \mathrm{cm}^{-1}$ axially leading to an elongated cloud at a distance of $1.5 \mathrm{~mm}$ from the chip surface. The MOT lasers and current through the U-wire are then turned off simultaneously in less than $100 \mu \mathrm{s}$ and the atoms are optically pumped into the $F=2, m_{F}=2$ ground state in $400 \mu \mathrm{s}$. A current of $4 \mathrm{~A}$ is then turned on through both $\mathrm{Z}$-wires on the chip and the atoms are caught in the potential produced by the wire and a $12 \mathrm{G}$ transverse bias field. The bias is ramped linearly from $12 \mathrm{G}$ to $30 \mathrm{G}$ over $500 \mathrm{~ms}$ to compress the cloud and achieve final trapping frequencies of $570 \mathrm{~Hz}$ radially and $11.2 \mathrm{~Hz}$ axially at a distance of $430 \mu \mathrm{m}$ from the surface. The lifetime of atoms in the magnetic trap is more than $30 \mathrm{~s}$, indicating that the vacuum is not adversely affected by the chip. At this point efficient evaporative cooling can begin.

The atomic cloud is evaporatively cooled through the BEC transition using a single logarithmic sweep of a radio frequency (RF) magnetic field from $13 \mathrm{MHz}$ to around $1 \mathrm{MHz}$ in $10.5 \mathrm{~s}$. In the two-wire trap this produces condensates of around $3 \times 10^{4}$ atoms. Larger condensates can be produced by further compressing the cloud into a trap formed by current through a single wire. Figure 4 shows absorption images of atom clouds following $15 \mathrm{~ms}$ free expansion after being released from a single wire magnetic trap. The three images show clouds after terminating the RF evaporation at $1080 \mathrm{kHz}, 1050 \mathrm{kHz}$ and $1010 \mathrm{kHz}$ respectively, in a 


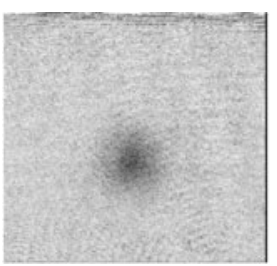

(a)

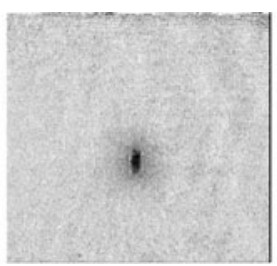

(b)

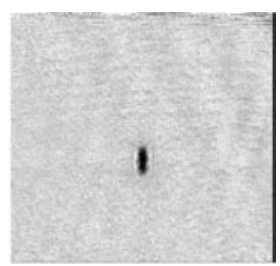

(c)

Figure 4. Absorption images after $15 \mathrm{~ms}$ free expansion of (a) a thermal cloud at $700 \mathrm{nK}$, (b) a partially condensed cloud at $450 \mathrm{nK}$, and (c) an almost pure BEC below $250 \mathrm{nK}$. A condensate forms below the critical temperature of about $500 \mathrm{nK}$. The chip wires are visible at the top of the images.

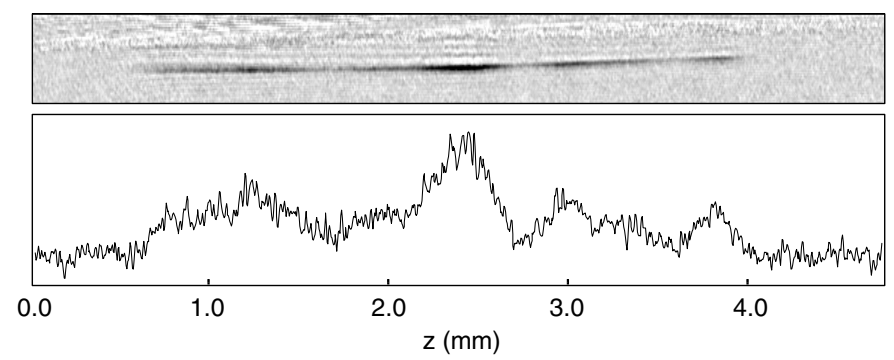

Figure 5. Absorption image (top) and cross-sectional profile (bottom) of a $4 \mu \mathrm{K}$ fragmented atom cloud prepared at a distance of $45 \mu \mathrm{m}$ from the chip. The trap was accelerated away from the surface for $5 \mathrm{~ms}$ before imaging.

trap with an offset field of $1.4 \mathrm{G}$. The trap forms $200 \mu \mathrm{m}$ from the chip surface with oscillation frequencies of $1100 \mathrm{~Hz}$ radially and $6 \mathrm{~Hz}$ axially. The condensate contains around $5 \times$ $10^{4}$ atoms and has a peak density of $4 \times 10^{14} \mathrm{~cm}^{-3}$. With our parameters the critical temperature for condensation is around $500 \mathrm{nK}$, which is reached with approximately $1.2 \times$ $10^{5}$ atoms.

\section{Fragmentation}

When cold atom clouds are brought close to current-carrying wires, the atomic distribution is seen to fragment into 'lumps' [13-15]. This is apparently due to microscopic deviations in the direction of current flow which produce small components of magnetic field parallel or antiparallel to the axis of the wire [13]. This effect may prove to be a limitation of current-carrying wire-based atom chips for applications such as atom interferometry.

We also observe fragmentation of cold clouds brought close to the chip surface. Figure 5 shows an absorption image and an averaged cross-sectional profile of a fragmented atom cloud after being accelerated away from the chip surface. The cloud was prepared at a distance of $45 \pm 5 \mu \mathrm{m}$ from a single wire at a temperature of $4 \mu \mathrm{K}$. At this distance the atoms could not be directly viewed in the trap due to light scattered from the gaps between the wires. Therefore the atoms were accelerated away from the surface for $5 \mathrm{~ms}$ by ramping up the current through the wire immediately before being imaged. Because of the short acceleration time and low cloud temperature and axial trapping frequency (nominally $2.9 \mathrm{~Hz}$ ), any redistribution of atoms in the axial direction in the displaced trap is not significant in the image. 
We observe larger structure in the atomic density profile than has been seen in other experiments - up to the millimetre scale. The cross-sectional profile also reveals shorter wavelength structure superposed on these larger fragments. This may be caused by slight deviations in the movement of the cutter during milling producing wires that are not perfectly straight - however we have no direct evidence of this as yet. The depth of the fragmenting potential is comparable to what has been seen above copper conductors [15].

Fragmentation appears to be highly dependent on the geometric and material properties of the conductors [7]. Our method of chip fabrication may be beneficial in this regard, as the high-purity solid metal foil should have superior conductor uniformity to electroplated wires. It remains to be seen how precisely wires can be patterned into a solid foil, and subsequently how this affects fragmentation. This topic will be investigated in future work. Regardless of any improvements in uniformity, our higher current-carrying capacity allows us to produce moderately tight traps further away from the conductors. This is important as fragmentation is seen to scale approximately as $\mathrm{e}^{-k y} / \sqrt{k y}$, where $k$ is the wavenumber of the current deviations [15].

\section{Conclusion}

We have demonstrated a novel method of producing atom chips suitable for the production and manipulation of Bose-Einstein condensates. Our chip is capable of sustaining higher currents than typical lithographically patterned chips, and can therefore produce deeper magnetic traps. This facilitates condensate production without sacrificing the versatility of the atom chip. The patterning of wires on our chip is currently at the $100 \mu \mathrm{m}$ scale but could be reduced using laser cutting to produce the insulating channels. Nonetheless, our technique has proven to be simple and reliable. Because of the higher current-carrying capacity of this set-up, we are able to produce moderately tight traps at distances greater than $100 \mu \mathrm{m}$ from the surface, where fragmentation effects become less important.

\section{Acknowledgments}

We are grateful to the Physics mechanical workshop at the University of Queensland, and in particular to Evan Jones for excellent technical work in manufacturing the chip. We also thank J Fortágh and T Campey for assistance with experiments. This work is supported by the Australian Research Council.

\section{References}

[1] Ott H, Fortagh J, Schlotterbeck G, Grossmann A and Zimmermann C 2001 Phys. Rev. Lett. 87230401

[2] Hänsel W, Hommelhoff P, Hänsch T W and Reichel J 2001 Nature 413498

[3] Folman R, Krüger P, Schmiedmayer J, Denschlag J and Henkel C 2002 Adv. At. Mol. Opt. Phys. 48263

[4] Hänsel W, Reichel J, Hommelhoff P and Hänsch T W 2001 Appl. Phys. B 7281

[5] Fortágh J, Ott H, Schlotterbeck G, Zimmermann C, Herzog B and Wharam D 2002 Appl. Phys. Lett. 811146

[6] Leanhardt A E, Chikkatur A P, Kielpinski D, Shin Y, Gustavson T L, Ketterle W and Pritchard D E 2002 Phys. Rev. Lett. 89040401

[7] Estéve J, Aussibal C, Schumm T, Figl C, Mailly D, Bouchoule I, Westbrook C and Aspect A 2004 Preprint physics/0403020

[8] Jones M P A, Vale C J, Sahagun D, Hall B V and Hinds E A 2003 Phys. Rev. Lett. 91080401

[9] Schneider S, Kasper S, vom Hagen Ch, Bartenstein M, Engeser B, Schumm T, Bar-Joseph I, Folman R, Feenstra L and Schmiedmayer J 2003 Phys. Rev. A 67023612 
[10] Weinstein J D and Libbrecht K G 1995 Phys. Rev. A 524004

[11] Hinds E A and Hughes I G 1999 J. Phys. D: Appl. Phys. 32 R119

[12] Hinds E A, Vale C J and Boshier M G 2001 Phys. Rev. Lett. 861462

[13] Kraft S, Günther A, Ott H, Wharam D, Zimmermann C and Fortágh J 2002 J. Phys. B: At. Mol. Opt. Phys. 35469

[14] Leanhardt A E, Shin Y, Chikkatur A P, Kielpinski D, Ketterle W and Pritchard D E 2003 Phys. Rev. Lett. 90100404

[15] Jones M P A, Vale C J, Sahagun D, Hall B V, Eberlein C C, Sauer B E and Hinds E A 2004 J. Phys. B: At. Mol. Opt. Phys. 37 L15 